\title{
Public transport versus private car GIS-based estimation of accessibility applied to the Tel Aviv metropolitan area
}

\author{
Itzhak Benenson • Karel Martens • Yodan Rofé • \\ Ariela Kwartler
}

Received: 7 July 2009 / Accepted: 1 April 2010 / Published online: 19 June 2010

(C) The Author(s) 2010. This article is published with open access at Springerlink.com

\begin{abstract}
The increasing interest in sustainable development has underlined the importance of accessibility as a key indicator to assess transport investments, urban policy, and urban form. From both the environmental and the equity component of sustainability, a comparison of accessibility by car versus public transport is of utmost importance. However, most studies in this direction have used rather rough estimates of travel time, especially by public transport. In this paper, we present Urban.Access, an ArcGIS extension for estimating car-based and transit-based accessibility to employment and other land uses. Urban.Access enables a detailed representation of travel times by transit and car and thus makes it possible to adequately compare accessibility levels by transport mode. The application of Urban.Access to the Tel Aviv metropolitan area shows that the gaps between car-based and transit-based accessibility are larger than those found in other studies. We argue that this is not the result of a poorer transit system, but rather of a more detailed description of travel by transit in the Urban.Access application. The larger gaps point to a greater need for adequate policy responses, both for reducing car dependence as well as for creating a more equitable
\end{abstract}

\footnotetext{
I. Benenson $(\varangle) \cdot$ A. Kwartler

Department of Geography and Human Environment, Tel Aviv University, Ramat Aviv, 69978 Tel Aviv, Israel

e-mail: bennya@post.tau.ac.il

K. Martens

Institute for Management Research, Radboud University Nijmegen, PO Box 9108, 6500 HK Nijmegen, The Netherlands

e-mail: k.martens@fm.ru.nl

Y. Rofé

Jacob Blaustein Institutes for Desert Research, Ben Gurion University of the Negev, 84990 Beer Sheva, Israel

e-mail: yrofe@bgu.ac.il
} 
transport system. Hence, we uphold that an adequate representation of transit travel times is more than a scientific matter-it is a matter of great social importance.

\section{JEL Classification Major R41 · Additional R48}

\section{Introduction: sustainable development and accessibility}

Increasingly, accessibility is identified as a key criterion to assess transport policies, as well as land use developments and urban service delivery policies (Bristow and Farrington et al. 2009). The recent emphasis in transport policy on accessibility is in part based on the long-standing understanding that transport is a derived need; that ultimately the accessibility provided by the transport system is what counts. Accessibility has also been identified as a performance indicator that can link land use and transport and thus provide a basis for more comprehensive, integrated policies that encompass coordinated action in both fields at once (Helling 1998).

The increasing interest in sustainable development has further underlined the importance of accessibility as a key indicator to assess urban form, the density and spatial distribution of people and activities, as well as urban policy. Accessibility, understood as the ability of people to reach and participate in activities (Garb and Levine 2002), is a valuable criterion for each of the three pillars of sustainable development: economic development, environmental quality, and social equity.

Accessibility is often viewed as a precondition for economic development, as it enables the exchange of people (labor) and goods (products), and hence an efficient functioning of the economy (Bruinsma and Nijkamp et al. 1990). From the perspective of environmental quality, attention is directed towards the differences between transport modes in terms of energy use and environmental externalities (Feitelson 2002). Here, it is used as a performance indicator to assess the differences in accessibility provided by more versus less energy-intensive and polluting modes of transport (Kwok and Yeh 2004). The social justice dimension of sustainable development, in turn, draws attention towards the distribution of benefits and burdens over members of society. Starting from transport as a derived need, accessibility is used in this context as an indicator of the extent to which all groups can participate in activities considered 'normal' to their society, such as access to employment and essential services (Farrington and Farrington 2005). In both cases, accessibility is a key policy indicator, and accessibility measures are necessary for adequate policy development.

In this paper, we present an accessibility measure that is especially useful to address the environmental and equity dimensions of sustainable development. Both dimensions require an accessibility measure with a strong comparative component between transport modes, although the emphasis of the comparison differs with regard to each dimension.

In case of the environmental dimension, the focus is on the degree of cardependence of a metropolitan area or part of it, given the externalities related to private car use vis-à-vis other modes of transport. When car accessibility is substantially higher than public transport accessibility, an area can be defined as car dependent and the car share in the modal split of an area is expected to be high, suggesting 
that transport-related negative externalities can be reduced through an improvement of accessibility by alternative modes of transport (Shen 1998; Blumenberg and Ong 2001; Hess 2005; Kawabata and Shen 2006; Kawabata 2009; Kwok and Yeh 2004). The relevant comparison is thus car versus public transport accessibility at the level of an area (e.g. neighborhood, census tract, or transport activity zone).

From an equity perspective, too, a comparison between accessibility for different transport modes is required (Martens 2009). Here, too, a comparison between carbased and public transport-based accessibility is important, because, on the one hand, car tends to provide substantially higher levels of accessibility in most contemporary urban areas, and, on the other hand, access to a car is not universal due to barriers inhibiting car ownership and use (costs, legal requirements and physical abilities). As for the environmental dimension, a comparison at the intra-area level is necessary, as it provides insight into the extent to which persons or households without access to a car have a comparable level of access as car-owning persons residing in the same area.

In the following, we develop accessibility measures that make it possible to carry out these comparisons. The paper is organized as follows. Section 2 provides a brief overview of existing measures of access and accessibility. In Sect. 3, we develop a comparative accessibility index, which can be used to assess accessibility from an environmental and equity perspective. Section 4 presents Urban.Access, an ArcGIS application that we have developed to determine car-based and transit-based accessibility in a detailed manner. In Sect. 5, Urban.Access is applied to analyze the gaps between car-based and transit-based accessibility in the Tel Aviv metropolitan area. The paper ends with a brief discussion of the developed GIS application and the advantages it offers over existing methods to measure transit-based accessibility.

\section{Accessibility measures}

In order to address transport-related environmental impacts and transport-related poverty through transportation planning and policy, it is necessary to assess the scale and quality of transport alternatives available to people. In the literature, accessibility measures have been suggested as the primary tool to gain insight into these issues. In this section, we provide a brief overview of the most important accessibility measures. Based on this overview, we outline in what way the approach presented in this paper adds to the existing body of knowledge.

A wide variety of accessibility measures has been developed over the past decades. An overview is provided by, amongst others, Handy and Niemeier (1997) and Geurs and Ritsema van Eck (2001). Geurs and van Wee (2004) distinguish four components that are of importance in measuring accessibility: transportation, land-use, temporal and individual. The transportation component describes the transport system expressed as the disutility for an individual to cover the distance between an origin and a destination using a specific transport mode. The land-use component consists of the distribution of various types of land-uses over space, as defined in terms of quantity (residential and employment density) and quality (level of employment, housing values or importance of services such as major hospitals or educational and cultural institutions). The temporal component reflects the temporal constraints, such as the 
availability of opportunities at different times of the day, and the time available for individuals to participate in certain activities (e.g. work, education, recreation). The individual component reflects the needs, abilities, and opportunities of individuals.

The extent to which these components are captured differs between accessibility measures. Geurs and van Wee (2004) distinguish between four types of accessibility measures:

- Infrastructure-based measures, which provide insight into the performance or service level of transport infrastructure (e.g. "the average travel speed on the road network').

- Location-based measures, which provide insight in the accessibility of locations (e.g. 'the number of jobs within 30 min travel from origin locations').

- Person-based measures, analyzing accessibility at the individual level taking into account personal possibilities and constraints (e.g. 'the number of activities in which an individual can participate at a given time').

- Utility-based measures, analyzing the (economic) benefits that people derive from access to the spatially distributed activities.

The majority of studies that compare access by car and by transit, as is required from the perspective used in this paper, use location-based measures. This preference may come as no surprise. Infrastructure-based measures are hardly relevant, as they focus on the quality of the transport network, but do not provide insight into accessibility levels experienced between different areas or groups of households within an area. Both person-based and utility-based measures, in turn, are highly dependent on data input and therefore difficult to apply at the level of a metropolitan area (but see Dong and Ben-Akiva et al. 2006).

Studies that apply location-based measures to assess the differential accessibility by car and transit include Shen (1998), Blumenberg and Ong (2001), Hess (2005), Kawabata and Shen (2006) and Kawabata (2009). These and comparable studies focus on accessibility to employment and apply relatively simple estimates of mode-based travel time to compare accessibility levels. In virtually all cases, travel time thresholds are used, rather than more complex gravity-based impedance functions. The spatial scale of analysis is usually rather large, with the typical study focusing on census tracts, neighborhoods or transport activity zones. The calculation of mode-based travel times is often based on data available from transport demand models and transit schedules. For car-based accessibility, this implies that delays due to congestion are often included, while for transit in-vehicle travel time is usually employed as the input to calculate transit-based accessibility. Other components of transit-related travel time, such as access and egress times, waiting times and transit transfer times, are hardly ever included in the calculations of total transit travel time or only by using very rough estimates.

Looking at the existing work on comparative accessibility analysis, three conclusions can be drawn. First, most accessibility measures applied in the literature cited above use rather rough estimates of travel time. They calculate public transit times at the level of zones or neighborhood. While this is sufficient for car accessibility, as the few minutes of extra drive time within the neighborhood are not crucial for accessibility, the walking distance to a transit stop is very crucial for public transport 
accessibility. So, one side of a neighborhood or a traffic zone can be well served by transit, while the other, $800 \mathrm{~m}$ away, is poorly served. Hence, detailed calculations are necessary at the address level to determine transit accessibility. The resulting data can then further be aggregated over the area and is likely to differ from average transit accessibility initially calculated at an aggregate zonal level. The difference in approach is important from a comparative perspective, as total transit travel times are heavily shaped by access and egress times, as well as by the assumptions regarding expected waiting time and number of transfers between transit lines that are allowed. A more accurate assessment of travel time by public transport will thus generate a more realistic picture of the accessibility gaps between car-less versus car-owning households. This may well shed a different light on the relatively small car-transit disparities found by, e.g., Hess (2005) and therefore result in different conclusions regarding the size of the accessibility gap. In the following, we will develop a method to comparatively assess transit and car travel times.

Second, most of the studies cited earlier provide a comparison of car-based and transit-based accessibility, but do not develop a practically applicable tool to carry out such assessments. As a result, the studies tend to remain in the academic realm, while comparative accessibility analysis is strongly called for in planning practice, for both environmental and equity reasons, as outlined in the Sect. 1.

Third, the authors carrying out comparative accessibility analysis primarily call for increasingly complex access measures (e.g. Doi and Kii et al. 2008). While there are solid theoretical grounds to include, for example, competition over jobs into accessibility measures (Kawabata 2003, p. 1653), we argue that for application in the context of transport and land use planning, simple measures are to be preferred over more complex measures. We see three reasons for this. First, while it has been acknowledged that most accessibility measures, with the exception perhaps of infrastructure-based measures, are comparative in nature, there has still been a tendency to develop measures that 'better' represent real-life accessibility levels. However, from the sustainability perspective, a perfect match between the particular estimates of accessibility and reallife accessibility levels is of limited importance. More important is the capability of such measures to identify the existing large disparities in accessibility levels. It is by no means certain that more comprehensive measures provide better estimates of these disparities or result in the identification of different areas or neighborhoods that experience substantial disparities. Second, we contend that comprehensive measures are at odds with the practice of transport planning, which tends to rely on relatively simple measures of car accessibility (notably, level-of-service indicators). In addition, the comprehensive measures are very difficult to use in land use decisions, as often very little is known about a projected development except its overall residential and employment densities and how it is connected to the street grid.

Given the tension between rigor and relevance, the latter is to be preferred in developing accessibility measures to address the environmental and equity dimensions of sustainability. This is especially prudent, given the complex and often inventive ways in which people adapt their travel habits to needs and constraints and the consequences for accessibility levels which could be derived from these strategies. What we are attempting here is to measure only the extent to which the land use-transportation system enables and supports an environmentally sound and equitable mode of travel. 
This is essentially captured by the comparison of car versus transit accessibility and the measures developed below that express the difference between them.

The method for comparative analysis of accessibility presented here differs in two respects from previous work in this direction. First, we present a practically applicable, GIS-based, tool that can be used in metropolitan areas throughout the world to assess car-based and transit-based accessibility (provided that the necessary data are available). Second, building on the GIS-based character of the application, we develop a technique to make more accurate estimates of transit-based accessibility, and, hence, are able to reveal existing accessibility gaps in a more detailed manner. The method is based in part on insights from previous studies into accessibility disparities (Kwok and Yeh 2004; Kawabata 2009), as well as from studies on public transport quality (Wu and Hine 2003; Wu and Murray 2005; Lao and Liu 2009).

It should be noted that the method presented here differs from comparable approaches that could be employed using standard travel forecasting methods (e.g. de Dios Ortuzar and Willumsen 2001) and software (such as TRANSCAD). Travel forecasting methods are first and foremost employed to determine the future demand for transport on each link of the (future) transport network. As such, optimal path algorithms are of key importance to determine the use of each transport link. While travel forecasting software could be employed to calculate car-based and transit-based accessibility levels, the use of optimal path algorithms makes this substantially more time-consuming. Our approach does not aim to forecast travel demand, but to assess the extent to which different locations can be accessed from each and every location in a study area. The calculation of an optimal path is not necessary for this purpose. As discussed in the following, travel speeds on the different components of the transport network can be derived from the results of transport models or based on data on actual speeds on the network. Note that we are looking into the structural inequalities built into the land-use and transportation system, as a first step in formulating policy to overcome them.

\section{Accessibility measure}

Like other studies into accessibility disparities, we develop a location-based measure of accessibility to urban functions and to employment. We start with the transport component and estimate access and service areas for a given type of transportation mode (car and transit). Given the dominance of the bus system in the Tel Aviv transit network, the analysis has been limited to the bus system only. The method has been developed in such a way that additional transit modes (the existing train lines or the future light rail line) can be incorporated into the analysis in the future.

In what follows, the variables related to public transportation have an index B (Bus) and the variables related to private cars have an index $\mathrm{C}(\mathrm{Car})$. The variables that regard trip origin have an index $\mathrm{O}$, while those related to the destination have an index $\mathrm{D}$.

\subsection{Definition of mode-based accessibility}

Following other studies, our measures of accessibility are based on the estimate of the travel time between $(\mathrm{O})$ rigin and (D)estination. We define them for a given transportation (M)ode: public (B)us, and private (C)ar. 
- Bus travel time (BTT):

$$
\begin{aligned}
& \text { BTT = Walk time from origin to a stop of Bus } 1 \\
& + \text { Waiting time of Bus } 1+\text { Travel time of Bus } 1 \\
& + \text { [Transfer walk time to Bus } 2+\text { Waiting } \\
& \text { time of Bus } 2+\text { Travel time of Bus } 2]+[\text { Transfer } \\
& \text { component related to additional buses }] \\
& \text { + Walk time from the final stop to destination. }
\end{aligned}
$$

The square brackets in the formula denote optional components. Noting that walking velocity is essentially lower than that of a car or bus, we assume that given the origin $\mathrm{O}$ and the destination $\mathrm{D}$, an agent always starts a trip at a bus stop relatively close to $\mathrm{O}$ and arrives at a bus stop relatively close to D. In what follows, we specify "relatively close" as a bus stop at $300 \mathrm{~m}$ air distance from $\mathrm{O}$ or D, i.e., within a 5- to 10-min walk from $\mathrm{O}$ or $\mathrm{D}$.

- Car travel time (CTT):

$\mathrm{CTT}=$ Walk time from origin to the parking place

+ Car trip time + Walk time from the

final parking place to destination

We assume that road congestion influences both BTT and CTT in the same proportion, that is, if BTT increases with the growth of congestion $20 \%$, similar increase is characteristic of CTT. This is in line with the current Tel Aviv situation, in which well-functioning bus lanes that circumvent congestion are virtually lacking.

- Access Area and Service Area:

Access Area: Given origin O, transportation mode M, and travel time $\tau$, let us define Mode Access Area, $\mathrm{MAA}_{O}(\tau)$, as the area containing all destinations $\mathrm{D}$ that can be reached from $\mathrm{O}$ with $\mathrm{M}$ during $\mathrm{MTT} \leq \tau$ (MTT $=$ Mode Travel Time).

Service Area: Given destination D, transportation mode M, and travel time $\tau$, let us define Mode Service Area, $\operatorname{MSA}_{D}(\tau)$, as the area containing all origins $\mathrm{O}$ from which given destination $\mathrm{D}$ can be reached with M during MTT $\leq \tau$.

While essentially the same calculation, the access area is more relevant to assess the relative accessibility from different residential areas, while the service area is useful to assess the accessibility to various employment and service centers. The former is especially relevant for the analysis of the equity component of accessibility, while both measures are relevant for assessing accessibility from the environmental perspective.

\subsection{Defining accessibility as the ratio of access or service areas}

In line with the goal of the paper and the literature (Kwok and Yeh 2004) we have developed two comparative accessibility measures. The measures define accessibility 
levels in terms of the gap between transit-based and car-based accessibility. More specifically, we have defined two accessibility measures that describe the disparities between transit-based and car-based accessibility: the Access Area ratio, and the Service Area ratio.

Given an origin O, we define the Bus to $\operatorname{Car}(B / C)$ Access Areas ratio as

$$
\operatorname{AA}_{O}(\tau)=\operatorname{BAA}_{O}(\tau) / \mathrm{CAA}_{O}(\tau)
$$

Given the destination D, we define the Bus to Car (B/C) Service Area ratio as

$$
\operatorname{SA}_{D}(\tau)=\operatorname{BSA}_{D}(\tau) / \operatorname{CSA}_{D}(\tau)
$$

Formulae (3) and (4) can be easily specified for a particular type k of destinations $\mathrm{D}_{\mathrm{k}}$ or origins $\mathrm{O}_{\mathrm{k}}$ and, further, towards including destination and origin capacities $\mathrm{D}_{k}$, Capacity, $\mathrm{O}_{\mathrm{k}}$, Capacity (for instance high-tech enterprises with destination capacity defined as a number of jobs, or low cost dwellings with origin capacity defined as number of apartments). The B/C Access Area ratio to destinations of type $\mathrm{k}$ can be defined as the sum of capacities of the destinations (e.g., the number of low wage jobs) that can be accessed during time $\tau$ with Bus and Car:

$$
\begin{aligned}
\operatorname{AA}_{\mathrm{O}, \mathrm{k}}(\tau)= & \Sigma_{\mathrm{Dk}}\left\{\mathrm{D}_{\mathrm{k}, \text { Capacity }} \mid \mathrm{D}_{\mathrm{k}} \in \mathrm{BAA}_{\mathrm{O}}(\tau)\right\} / \Sigma_{\mathrm{Dk}} \\
& \times\left\{\mathrm{D}_{\mathrm{k}, \text { Capacity }} \mid \mathrm{D}_{\mathrm{k}} \in \mathrm{CAA}_{\mathrm{O}}(\tau)\right\}
\end{aligned}
$$

Likewise, B/C Service Area ratio for origins of type k can be defined as the sum of capacities of the origins (e.g., number of apartments in low income neighborhoods) that can be accessed during time $\tau$ with Bus and Car, respectively:

$$
\begin{aligned}
\operatorname{SA}_{\mathrm{D}, \mathrm{k}}(\tau)= & \Sigma_{\mathrm{Ok}}\left\{\mathrm{O}_{\mathrm{k}, \text { Capacity }} \mid \mathrm{O}_{\mathrm{k}} \in \mathrm{BSA}_{\mathrm{D}}(\tau)\right\} / \Sigma_{\mathrm{Ok}} \\
& \times\left\{\mathrm{O}_{\mathrm{K}, \text { Capacity }} \mid \mathrm{O}_{\mathrm{k}} \in \mathrm{CSA}_{\mathrm{D}}(\tau)\right\}
\end{aligned}
$$

The sum of the nominators of (5)-(6) is the overall capacity of the access/service areas estimated for the bus mode and the sum in the denominator is the overall capacity of the access/service areas estimated for the car mode.

The formulae (3)-(6) result in index varying between 0 and 1 or, alternatively, between 0 and $100 \%$. The interpretation of this index is straightforward, even for the more complex formulae (5) and (6). For instance, for destinations of type $\mathrm{j}$ (job), an outcome like $\mathrm{AA}_{\mathrm{O}},{ }_{j}(60 \mathrm{~min})=0.3$ would mean that "within $60 \mathrm{~min}$ of travel, bus users have access to $30 \%$ of jobs compared to car users". Likewise, for origins of type 1 (low-cost apartments), $\mathrm{SA}_{D, l}(60 \mathrm{~min})=0.3$ would mean that "the number of bus users living in a low-cost apartment that can reach the selected destination during $60 \mathrm{~min}$ travel is $30 \%$ compared to the number of car users that can reach that destination".

Due to lack of space, we only apply formulae (3) and (5) in the remainder of the paper, and estimate Access Area ratio (3) for all urban land uses taken together, as well 
as Job Access Ratio according to (5), in the latter case accounting for job capacity in terms of total number of jobs in the traffic area zone.

\section{Urban.Access as a tool for estimating accessibility}

To implement the aforementioned framework we have developed Urban.Access, a GIS application. Urban.Access is a Visual Basic for Applications (VBA) program developed by us within the ArcGIS environment for estimating transit-based Access and Service Areas. The estimates of transit accessibility obtained with Urban.Access are further combined, with the help of ArcGIS Model Builder, with the estimates of car-based accessibility obtained with the ArcGIS Network Analyst.

Urban.Access makes it possible to construct car and transit access and service areas for a chosen time frame from/to any area in a metropolitan area. Based on the access/service areas, it enables calculating car-based and transit-based accessibility to different types of land uses or set of locations. It furthermore generates indices of accessibility, as (3) and (4), and indices of accessibility for origins/destinations of particular types accounting for their capacities, as (5) and (6), provided the required data are available.

The Urban.Access GeoDatabase consists of

- A layer of roads with the attributes sufficient for constructing a network, and, if available, a table of turn restrictions. The average speed of the private car and public transport should be provided for each road link, for peak and off-peak hours, or at the higher temporal resolution.

- A layer of transit stops and a layer of the transit lines: each transit line should be related to the links of the road network it passes. The stops of different lines located at the same point in space are considered as different stops. Each transit stop is related to its line.

- A table of transit departure and arrival times, by line.

- Optional: layers of urban land uses and layers of origin/destinations of various types with capacities given.

These layers and table are necessary for estimating transit access/service areas and accessibility as given in (3) and (4); the optional layer of the land-uses or of origin/ destinations of a given type enables estimating accessibility by types and in respect to origin/destination capacities, as given by (5) and (6).

Important to note, that based on the layers of stops and lines, at the preprocessing stage, Urban.Access constructs a table of transfers. This table contains all pairs of stops at a distance below the "maximum transfer distance" (a pre-defined maximum walking distance, here set at $500 \mathrm{~m}$ air distance) and the distance between these stops. It is used for estimating possible travelers' transfers between different transit lines. For the Tel Aviv bus network, with its 350 basic bus lines, the majority of which have several variants depending on the time of the day and the day of the week (ca. 2,300 variants altogether), and ca. 65,000 stops at ca. 10,000 different locations, the table of transfers contains ca. 5,000,000 records. 


\subsection{Parameter setting in Urban.Access}

To run Urban.Access, the user has to define a number of parameters:

- Day of the week: This is necessary, as bus timetables vary by day of the week.

- Trip start time: In case of an access area, this parameter defines the time an agent arrives to the initial bus stop/parking place and starts waiting for a bus or driving. In case a service area is calculated, this parameter denotes the time an agent aims at arriving to a stop within the area of destination (and continues to the final destination by foot).

- Maximum waiting time at initial stop: If an agent waits at the start for longer than the predefined maximum time, the trip is canceled. This parameter represents the knowledge of agents of the bus time-schedule at the initial stop.

- Walking speed: This is employed for estimating the time necessary for transfer between bus stops, as well as the access time to the initial bus stop and egress time to the destination. In the current version of Urban.Access, only transfer time between lines is incorporated in the calculations. For reasons of simplicity, access and egress times are currently assumed to be equal for the bus and car modes.

- Access or egress walking distance: The maximum distance a person is willing to walk to the first and from the last bus stop.

- Maximum total travel time (i.e. travel time threshold): The maximal allowed travel time, excluding the time necessary to access the car/transit system or to walk to the final destination from the car park/final bus stop.

- Maximum number of transfers between bus lines: 0, 1, or 2. In the paper, we compare the results for 0 and 1 transfer.

- The user has to choose if either Access Area or Service Area is estimated.

In the results presented below, we examine the effects of maximum travel time on accessibility. More research is necessary to examine the sensitivity of results to the effect of walking time to the transport mode of choice as well as to the variations in numerical values of parameters.

It is important to note that Urban.Access is based on the Network Analyst extension of ArcGIS and employs its component which calculates "Network Service Area" for the car mode. The popular ArcGIS software was chosen to fit the software choice of the Tel Aviv Municipality. The use of advanced transportation software, such as TransCAD, would demand reprogramming of the application, but could merit from TransCAD's built-in abilities, such as its ability to find the optimal bus travel path between two stops.

\subsection{Applying Urban.Access for estimating access areas}

Figure 1 provides an example of the results generated by the Urban.Access application. It gives the access area for the Tel Aviv University bus terminal, at 0700 hours on Monday, for a travel time threshold of $45 \mathrm{~min}$, a maximum of one transfer, and a maximum walking distance to the initial bus stop of $300 \mathrm{~m}$ air distance.

The overall bus and car access areas, presented in Fig. 1, are 87 and $796 \mathrm{~km}^{2}$ for the overall area, and 64 and $273 \mathrm{~km}^{2}$ for the urban land-uses. Their comparison enables 

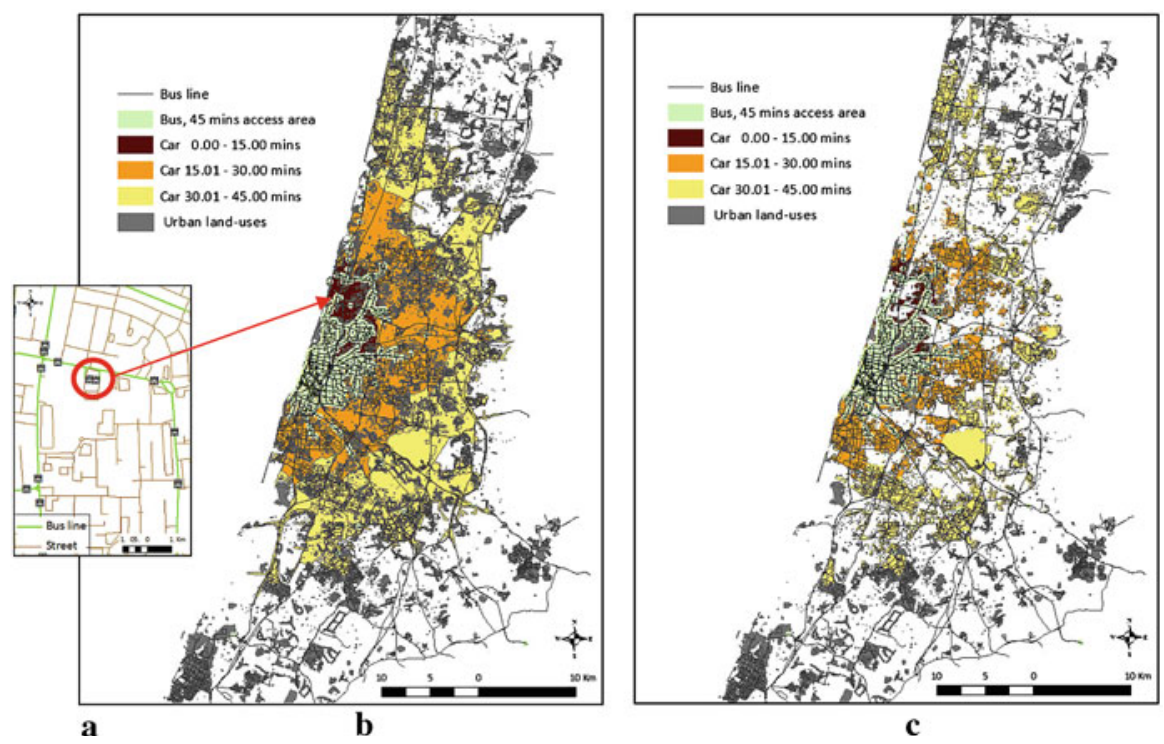

Fig. 1 a All bus stops in the traffic zone containing the Tel Aviv University bus terminal (circle); b 45- min bus and car access areas, the latter presented by 15-min rings; and c 45-min bus and car access areas limited to the layer of urban land uses

estimation of the transit-to-car Access Area index ( $\mathrm{AA}_{O}(\tau)$ index) for a 45-min travel time threshold (excluding the initial pedestrian walks):

$$
\begin{gathered}
\mathrm{AA}_{O}(45 \mathrm{~min})=87 \mathrm{~km}^{2} / 796 \mathrm{~km}^{2}=0.11 \text { (for overall area) } \\
\mathrm{AA}_{O}(45 \mathrm{~min})=64 \mathrm{~km}^{2} / 273 \mathrm{~km}^{2}=0.23 \text { (for urban land-uses) }
\end{gathered}
$$

Urban.Access thus shows that transit-based accessibility of Tel Aviv University is nearly ten times as poor as car-based accessibility when all land in the metropolitan area is taken into account, and over four times as poor when only urban land uses are taken into account.

\section{Analysis of accessibility in tel aviv metropolitan area}

We have applied Urban.Access to analyze the accessibility gaps in the Tel Aviv metropolitan area, for both urban land uses and employment. Below, we present the first results of this analysis. First, we turn to the overall figures, and then discuss the variation over the metropolitan area.

\subsection{Overall accessibility gaps}

The Urban.Access application makes it possible to estimate accessibility for any partition of the urban area, thus providing insight into the size of the gaps between 
Table 1 Access Area indices (AA, Urbanlanduses $(\tau)$ indices) for urban land-use destinations as dependent on travel time threshold and time of day (0700 vs. 1200 hours)

\begin{tabular}{|c|c|c|c|c|c|c|c|c|c|c|}
\hline \multirow{2}{*}{$\begin{array}{l}\text { Travel time threshold } \\
\text { Time of day (hours) }\end{array}$} & \multicolumn{2}{|c|}{$30 \mathrm{~min}$} & \multicolumn{2}{|c|}{$40 \mathrm{~min}$} & \multicolumn{2}{|c|}{$50 \mathrm{~min}$} & \multicolumn{2}{|c|}{$60 \mathrm{~min}$} & \multicolumn{2}{|c|}{ Average } \\
\hline & 0700 & 1200 & 0700 & 1200 & 0700 & 1200 & 0700 & 1200 & 0700 & 1200 \\
\hline Direct trip & 0.069 & 0.047 & 0.057 & 0.041 & 0.052 & 0.040 & 0.048 & 0.040 & 0.057 & 0.042 \\
\hline One transfer & 0.167 & 0.099 & 0.167 & 0.110 & 0.186 & 0.127 & 0.206 & 0.153 & 0.182 & 0.122 \\
\hline One transfer/Direct trip & 2.420 & 2.106 & 2.930 & 2.683 & 3.577 & 3.175 & 4.292 & 3.825 & 3.193 & 2.905 \\
\hline
\end{tabular}

Lower numbers point at larger gaps between car-based and transit-based accessibility

transit-based and car-based accessibility over the whole metropolitan area. This enables a comparison between metropolitan areas, as well as an assessment of the general impacts of specific policy interventions. Here, we present the results of Urban.Access regarding the average accessibility gap for urban land uses and for employment, for both peak and off-peak hours, for direct trips and for trips with one transfer. ${ }^{1}$

The accessibility gap for urban land uses is presented in Table 1. This roughly estimates access to residential and industrial areas, as well as leisure areas and services, and thus provides an indicator of the general level of accessibility. As might be expected, the variation of accessibility is very high: the coefficient of variance $(\mathrm{CV}=\mathrm{STD} \times 100 \% /$ Mean $)$ is about $80-90 \%$ for each of the values presented in Tables 1 and $2 .^{2}$

Table 1 clearly shows that transit-based accessibility is substantially lower than car-based accessibility. During the peak hours, transit serves about $6 \%$ of the total amount of urban land uses served by the car, when only direct transit trips are allowed, and about $18 \%$ in case of one transfer. During off-peak hours, these values decrease to about 4 and $12 \%$, respectively. These differences are a direct consequence of higher bus frequencies and line density in the peak hours, resulting in lower waiting times at the initial bus stop and lower transfer times, as well as lower car travel times outside peak hours.

The data furthermore show that, if no transit transfer is allowed, the Access Area index $\left(\mathrm{AA}_{O}(\tau)\right)$ does not improve with a growth in the travel time threshold, neither in peak nor in off-peak hours; in fact it actually degenerates. In contrast, when one transfer is allowed, the index slowly but continuously increases, from a value of 17 to $21 \%$ for a trip during the peak hours and from 10 to $15 \%$ during off-peak hours. These findings can be linked to the connectivity of the bus line network and underline its importance for transit-based accessibility.

Table 2 shows the results of the analysis of accessibility to employment in the metropolitan area. Like in the case of all urban land uses, there is a substantial gap between transit-based and car-based accessibility to employment. On average, transit

\footnotetext{
1 Almost all the areas in Tel Aviv metropolitan area can be reached with one transfer and the difference between the accessibility with one versus two transfers is insignificant.

2 The CVs of TAZ accessibility for all combinations of conditions presented in Tables 1 and 2 always vary within the 70-90\% interval for the direct trip and within the 40-60\% interval for one transfer and, by this reason, we do not include them into the tables.
} 
Table 2 Access Area indices (AA $O$, Employment $(\tau)$ indices), for employment destinations, as dependent on travel time threshold and time of day (0700 vs. 1200 hours)

\begin{tabular}{|c|c|c|c|c|c|c|c|c|c|c|}
\hline \multirow{2}{*}{$\begin{array}{l}\text { Travel time threshold } \\
\text { Time of day (hours) }\end{array}$} & \multicolumn{2}{|c|}{$30 \mathrm{~min}$} & \multicolumn{2}{|c|}{$40 \mathrm{~min}$} & \multicolumn{2}{|c|}{$50 \mathrm{~min}$} & \multicolumn{2}{|c|}{$60 \mathrm{~min}$} & \multicolumn{2}{|c|}{ Average } \\
\hline & 0700 & 1200 & 0700 & 1200 & 0700 & 1200 & 0700 & 1200 & 0700 & 1200 \\
\hline Direct trip & 0.119 & 0.083 & 0.102 & 0.077 & 0.102 & 0.080 & 0.106 & 0.086 & 0.107 & 0.082 \\
\hline One transfer & 0.263 & 0.169 & 0.266 & 0.193 & 0.308 & 0.231 & 0.356 & 0.287 & 0.298 & 0.220 \\
\hline One transfer/Direct trip & 2.210 & 2.036 & 2.608 & 2.506 & 3.020 & 2.888 & 3.358 & 3.337 & 2.785 & 2.683 \\
\hline
\end{tabular}

Lower numbers point to larger gaps between car-based and transit-based accessibility

travelers can directly reach about $11 \%$ of employment opportunities available to the car user at peak hours, and only $8 \%$ during off-peak hours. A transfer almost triples the accessibility ratio: to $30 \%$ during peak hours and to $22 \%$ during off-peak hours. However, even in case a transfer is allowed, the transit accessibility for employment is three to four times lower than that with the car. Just as in the case of accessibility to urban land uses, the direct accessibility does not depend on the travel time threshold, while transit accessibility grows from 26 to $36 \%$ during peak hours and from 17 to $29 \%$ during off-peak hours, with an increase of the threshold from 30 to $60 \mathrm{~min}$.

Note that according to Tables 1 and 2, job accessibility is, on average, about two times higher than urban area accessibility, for both a direct trip and a trip with one transfer. This reflects the fact that the transit network tends to be organized and scheduled towards major centers of employment, especially older inner city areas, while less intensively used routes, such as between different residential areas, tend to be poorly served by the transit system. This observation is further strengthened by the fact that, with an increasing travel time threshold, the difference between access to employment and access to all urban land grows.

\subsection{Differences in accessibility gaps in the metropolitan area}

The results presented above provide only a general picture of the accessibility gaps in the Tel Aviv metropolitan area. To gain further insight into the gaps, we present the GIS maps that Urban.Access generates. For reasons of space, we limit the analysis to accessibility to employment.

Figure 2 presents typical maps generated by Urban.Access. They present the Access Area indices for employment, averaged over the distance from the city core and aggregated at the level of transport activity zones (TAZs) at peak hours. Note that the use of the ratio between transit-based and car-based accessibility areas at least partially eliminates the problem of the decrease in accessibility level close to the boundary of a study area, due to the fact that the areas outside the studied region are not included in the calculations. In our case both the car and bus access/service areas are truncated by the studied area and by using their ratio the effect of the situation outside the study area is largely neutralized.

According to the map, relative accessibility to employment gradually decreases from the metropolitan core to periphery. If no bus transfer is allowed, the Access Area 


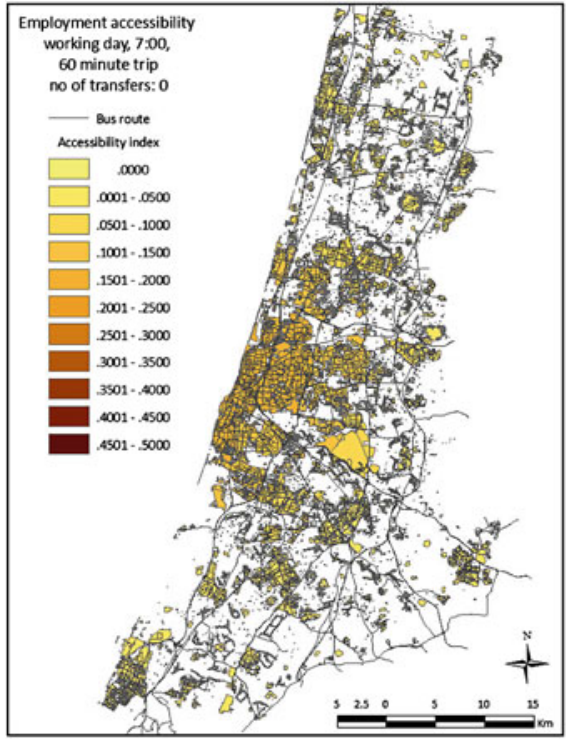

$\mathbf{a}$

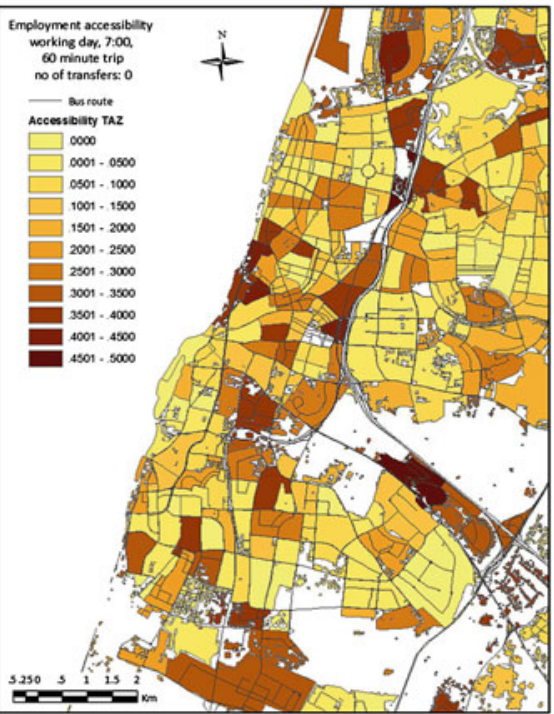

c

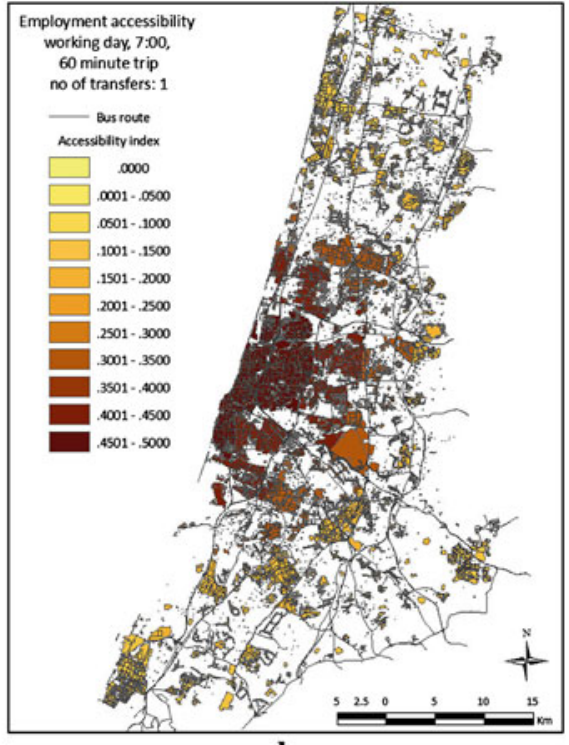

b

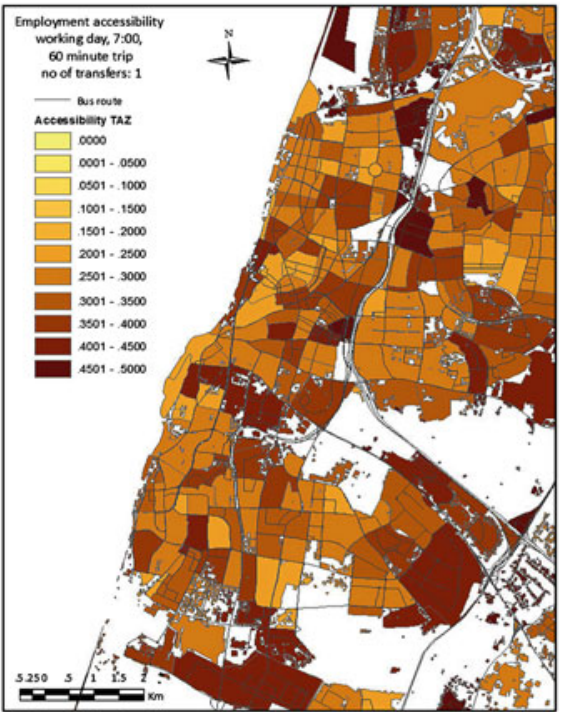

d

Fig. 2 Access Area index for employment averaged by the distance from the metropolitan core (a), (b), and at the resolution of transport activity zones (TAZs) within the metropolitan core (c), (d). For peak hours (0700 hours) and travel time threshold of $60 \mathrm{~min}$. Maps a and $\mathbf{c}$ represent employment accessibility for direct transit trips; $\mathbf{b}$ and $\mathbf{d}$ for transit trips that include one transfer. A value of $0.05-0.10$ implies that transit-based accessibility is between 5 and $10 \%$ of car-based accessibility

index decreases from $0.15-0.10$ to $0.07-0.03$; for the trips with one transfer the index decreases from $0.50-0.40$ to $0.20-0.10$. In the same time, large part of the TAZs in the metropolitan area show large accessibility gap, especially if no bus 
Table 3 Total number and share of TAZs and population by level of Access Area ratio for employment, for peak hours (0700 hours) and travel time threshold of $60 \mathrm{~min}$

\begin{tabular}{lccrrr}
\hline \multirow{2}{*}{ Access Area ratio } & \multicolumn{2}{c}{ Share of TAZs (\%) } & & \multicolumn{2}{c}{ Share of total population (\%) } \\
\cline { 2 - 3 } & Direct & Transfer & & Direct & Transfer \\
\hline $0.000-0.10$ & 53 & 17 & & $63.3 \%$ & $30.3 \%$ \\
$0.101-0.20$ & 34 & 10 & & $28.9 \%$ & $13.8 \%$ \\
$0.201-0.30$ & 12 & 9 & & $7.8 \%$ & $8.0 \%$ \\
$0.301-0.40$ & 1 & 11 & & $0.0 \%$ & $8.9 \%$ \\
$0.401-0.50$ & 0 & 23 & & $0.0 \%$ & $21.6 \%$ \\
$0.501-1.00$ & 0 & 30 & & $0.0 \%$ & $17.5 \%$ \\
Total & $100 \%$ & $100 \%$ & & $100.0 \%$ & $100.0 \%$ \\
\hline
\end{tabular}

transfer is allowed: For more than half of the TAZ, transit users have direct access to less than $10 \%$ of the employment that is accessible by car from the same area. The trips with one transfer provide essentially higher accessibility than the direct trips, but yet for half of the areas transit users have access to less than $40 \%$ of the employment compared to the car mode (Table 3). This holds especially true for the areas located in the north, on the eastern border, and in the south-east of the metropolitan area. The population in these areas accounts for $60 \%$ of the total population of the metropolitan area (Table 3). Interestingly enough, the metropolitan center (along the coast, recognizable by the small size of the TAZs) does not show the smallest accessibility gaps. Instead, the areas with a relatively small gap are located along major transit corridors, served by relatively fast and frequent bus services.

The accessibility patterns for direct trips and one transfer are basically similar. Large gaps can be found in the peripheral areas, as well as in a number of central city areas, especially for the direct trip (Fig. 2; Table 3). The overall picture does improve when one transfer is allowed, as a substantial share of the TAZs is now fairly well served by transit (Fig. 2; Table 3). However, even in this case, only $30 \%$ of TAZ and $18 \%$ of the population score higher than $50 \%$, and only $3 \%$ of the TAZs and population scores higher than $60 \%$. This is in contrast to the situation in some Asian cities, where transit-based accessibility is actually better is some areas than car-based accessibility (see e.g. Kwok and Yeh 2004). Note that these findings, presented for a 60- min travel time threshold and peak hours only, remain valid for other travel time thresholds and times of day. Indeed, the correlation between the values of accessibility for time thresholds of 30, 40, 50, and $60 \mathrm{~min}$ and between the values of direct and one transfer accessibility is very high and varies between $0.85-0.95$. For a large share of the TAZs, the Access Area ratio hardly depends on the travel time threshold or whether the traveler prefers a direct line or is prepared to make a transfer.

\section{Discussion}

The results found for the Tel Aviv metropolitan area show large gaps between carbased and transit-based accessibility, which are similar to those found in USA, which 
is notorious for its poor transit network. For instance, Hess (2005) finds a car/transit job accessibility ratio of 1.7-8.2. In our terms, this amounts to an Access Area value of $12-59 \%$. Based on the Urban.Access application, we find even lower values for many areas than the lowest value observed by Hess. In our opinion, this is not the result of a poorer transit system, but rather of a more detailed description of travel by transit in the Urban.Access application. The results found by Blumenberg and Ong (2001), for two areas in the San Francisco area seem, at least at first sight, more comparable with the findings for Tel Aviv, with values, in our terms, of 2-19\% for access to low-wage, feminized, jobs. The advantage of our relative view of accessibility is that these figures can be easily compared to those found for Tel Aviv, despite the fact that they capture accessibility to only part of the total employment.

Given the counter-intuitive finding that the results for Tel Aviv, with its relatively dense bus network, are largely comparable to those found in US cities, we conclude that a more detailed representation of travel by transit results in larger accessibility gaps. These large gaps can be ascribed to the distinction between direct trips and trips with transfers, to a detailed analysis of transit travel time at the level of individual addresses, and to the inclusion of both the estimated waiting time at the outset of a trip, and the walking and waiting times related to (bus) transfers, in the accessibility index.

The large gaps found in the analysis point to a larger than previously estimated automobile dependence, particularly in outlying areas of the Tel Aviv metropolitan area. These patterns are non-sustainable from the environmental point of view, as they increase the need to drive cars in order to gain access to services and employment, and result in greater inequity, as those dependent on public transit for access find themselves with less options. Thus there is a greater need than previously thought for adequate policy responses to redress this imbalance. In the short term, dedicated bus lanes on congested roads will improve the relative accessibility of transit users, by shortening their travel time while possibly increasing travel times for car-users. In the longer term, the development of more rapid mass transit using its own right of way and concentrating development around it, will allow a large share of development to concentrate in zones where the ratio between transit and car accessibility is high.

Hence, an adequate representation of the gap between transit and car accessibility is more than a scientific matter-it is a matter of great environmental and social importance.

Open Access This article is distributed under the terms of the Creative Commons Attribution Noncommercial License which permits any noncommercial use, distribution, and reproduction in any medium, provided the original author(s) and source are credited.

\section{References}

Blumenberg EA, Ong P (2001) Cars, buses, and jobs: welfare participants and employment access in Los Angeles. Transp Res Record J Transp Res Board 1756:22-31

Bristow G, Farrington J et al (2009) Developing an evaluation framework for crosscutting policy goals: the Accessibility Policy Assessment Tool. Environ Plan A 41(1):48-62 
Bruinsma FR, Nijkamp P et al (1990) Infrastructure and metropolitan development in an international perspective: survey and methodological exploration. Faculteit der Economische Wetenschappen en Econometrie/Vrije Universiteit Amsterdam, Amsterdam

de Dios Ortuzar J, Willumsen LG (2001) Modelling transport. Wiley, Chichester

Doi K, Kii M et al (2008) An integrated evaluation method of accessibility, quality of life, and social interaction. Environ Plan B Plan Des 35(6):1098-1116

Dong X, Ben-Akiva ME et al (2006) Moving from trip-based to activity-based measures of accessibility. Transp Res A Policy Pract 40(2):163-180

Farrington J, Farrington C (2005) Rural accessibility, social inclusion and social justice: towards conceptualisation. J Transp Geogr SPEC ISS 13(1):1-12

Feitelson E (2002) Introducing environmental equity dimensions into the sustainable transport discourse: issues and pitfalls. Transp Res D Trans Environ 7(2):99-118

Garb Y, Levine J (2002) Congestion pricing's conditional promise: promotion of accessibility or mobility? Transp Policy 9(3):179-188

Geurs KT, Ritsema van Eck JR (2001) Accessibility measures: review and applications. Evaluation of accessibility impacts of land-use transport scenario's, and related social and economic impacts. RIVM, National Institute of Public Health and the Environment (NL), Bilthoven

Geurs KT, van Wee B (2004) Accessibility evaluation of land-use and transport strategies: review and research directions. J Transp Geogr 12(2):127-140

Handy SL, Niemeier DA (1997) Measuring accessibility: an exploration of issues and alternatives. Environ Plan A 29(7):1175-1194

Helling A (1998) Changing intra-metropolitan accessibility in the U.S.: evidence from Atlanta. Prog Plan 49(2):55-107

Hess DB (2005) Access to employment for adults in poverty in the Buffalo-Niagara region. Urban Stud 42(7):1177-1200

Kawabata M (2003) Job access and employment among low-skilled autoless workers in US metropolitan areas. Environ Plan A 35:1651-1668

Kawabata M (2009) Spatiotemporal dimensions of modal accessibility disparity in Boston and San Francisco. Environ Plan A 41(1):183-198

Kawabata M, Shen Q (2006) Job accessibility as an indicator of auto-oriented urban structure: a comparison of Boston and Los Angeles with Tokyo. Environ Plan B Plan Des 33(1):115-130

Kwok RCW, Yeh AGO (2004) The use of modal accessibility gap as an indicator for sustainable transport development. Environ Plan A 36(5):921-936

Lao Y, Liu L (2009) Performance evaluation of bus lines with data envelopment analysis and geographic information systems. Comput Environ Urban Syst 33(4):247-255

Martens K (2009) Justice in Transport: applying Walzer's 'Spheres of Justice' to the transport sector. Paper presented at the 88th Annual Meeting of the Transportation Research Board, 11-15 January 2009, Washington DC, USA

Shen Q (1998) Location characteristics of inner-city neighborhoods and employment accessibility of lowwage workers. Environ Plan B Plan Des 25(3):345-365

Wu BM, Hine JP (2003) A PTAL approach to measuring changes in bus service accessibility. Transp Policy 10(4):307-320

Wu CS, Murray AT (2005) Optimizing public transit quality and system access: the multiple-route, maximal covering/shortest-path problem. Environ Plan B Plan Des 32(2):163-178 